\title{
Diagnostic value and prognostic evaluation of Presepsin for sepsis in an emergency department
}

\author{
Bo Liu, Yun-Xia Chen, Qin Yin, Yun-Zhou Zhao and Chun-Sheng Li
}

\begin{abstract}
Introduction: Presepsin levels are known to be increased in sepsis. The aim of this study was to evaluate the early diagnostic and prognostic value of Presepsin compared with procalcitonin (PCT), Mortality in Emergency Department Sepsis (MEDS) score and Acute Physiology and Chronic Health Evaluation II (APACHE II) score in septic patients in an emergency department (ED) and to investigate Presepsin as a new biomarker of sepsis.

Methods: This study enrolled 859 consecutive patients with at least two diagnostic criteria for systemic inflammatory response syndrome (SIRS) who were admitted to Beijing Chao-yang Hospital ED from December 2011 to October 2012, and 100 age-matched healthy controls. Patients were stratified into four groups: SIRS, sepsis, severe sepsis, and septic shock. Plasma Presepsin and serum PCT were measured, and MEDS score and APACHE II score were calculated at enrollment. Comparisons were analyzed using the Kruskal-Wallis and Mann-Whitney $U$ tests.

Results: On admission, the median levels of plasma Presepsin increased with sepsis severity. The areas under the receiver operating characteristic (AUC) curves of Presepsin were greater than those of PCT in diagnosing sepsis, and predicting severe sepsis and septic shock. The AUC of Presepsin for predicting 28-day mortality in septic patients was slightly lower than that of PCT, MEDS score and APACHE II score. The AUC of a combination of Presepsin and MEDS score or APACHE II score was significantly higher than that of MEDS score or APACHE II score alone in predicting severe sepsis, and was markedly higher than that of Presepsin alone in predicting septic shock and 28-day mortality in septic patients, respectively. Plasma Presepsin levels in septic patients were significantly higher in non-survivors than in survivors at 28 days' follow-up. Presepsin, MEDS score and APACHE II score were found to be independent predictors of severe sepsis, septic shock and 28-day mortality in septic patients. The levels of plasma Presepsin were positively correlated with PCT, MEDS score and APACHE II score in every septic group.
\end{abstract}

Conclusion: Presepsin is a valuable biomarker for early diagnosis of sepsis, risk stratification, and evaluation of prognosis in septic patients in the ED.

\section{Introduction}

Sepsis still represents a major cause of morbidity and mortality in critically ill patients despite the use of modern antibiotics and resuscitation therapies [1]. There is a lack of early diagnosis and timely intervention for sepsis in the emergency department (ED), and recent interest has focused on biomarkers for early diagnosis, risk stratification, and evaluation of prognosis of sepsis.

CD14, the high-affinity receptor for lipopolysaccharide/ lipopolysaccharide binding protein complexes, is a glycoprotein expressed in macrophage, monocyte, and granulocyte

\footnotetext{
* Correspondence: Icscyyy@163.com

Emergency Department, Beijing Chao-yang Hospital, Capital Medical University, 8\# Worker's Stadium South Road, Beijing, Chao-yang District 100020, China
}

cells and their cell membranes [2]. The lipopolysaccharide-lipopolysaccharide binding protein-CD14 complex is released into circulation by shedding of CD14 from the cell membrane, yielding soluble CD14. Presepsin (soluble CD14-ST), a novel biomarker for diagnosing sepsis, is a subtype of soluble CD14, and is a $13 \mathrm{kDa}$ protein that is a truncated N-terminal fragment of CD14 [3].

Although some clinical studies confirmed that plasma presepsin levels were significantly increased in septic patients, and were positively correlated with the severity of sepsis, the sample sizes were relatively small [4-9], and these findings have not yet been corroborated in a large number of septic patients in the ED. The aim of this study was to investigate the clinical value of presepsin in early diagnosis, risk stratification and prognostic evaluation of

\section{Biomed Central}


sepsis in a large sample of septic patients in a hospital ED, and to compare it with the prognostic value of procalcitonin (PCT), the Mortality in Emergency Department Sepsis (MEDS) score and the Acute Physiology and Chronic Health Evaluation (APACHE) II score.

\section{Methods}

\section{Patient inclusion and exclusion criteria}

This prospective study was conducted in the ED of Beijing Chao-yang Hospital, a university teaching hospital with approximately 240,000 to 260,000 ED admissions per year. From December 2011 to October 2012, consecutive patients who fulfilled the criteria for sepsis as defined by the American College of Chest Physicians/ Society of Critical Care Medicine (ACCP/SCCM) were enrolled [10]. Patients were classified at the time of enrollment as having sterile systemic inflammatory response syndrome (SIRS), sepsis, severe sepsis and septic shock, according to ACCP/SCCM criteria [10].

Exclusion criteria were as follows: $<18$ years old, terminal stage of disease (malignant cancer of any type, acquired immunodeficiency syndrome, end-stage liver or renal disease), and the patient or relatives did not consent to inclusion. Finally, after excluding 92 patients who did not meet the inclusion criteria and 49 patients who were lost to follow-up, 859 patients were enrolled and were followed for 28 days or until death. At the same time, 100 age-matched healthy individuals were enrolled as controls. This study was approved by the Beijing Chao-yang Hospital Ethics Committee. Written informed consent was obtained from every subject.

\section{Establishment of infection}

The infections of different diseases in our study were clinically established on the basis of clinical features, laboratory findings, and imaging tests according to criteria of the International Sepsis Forum Consensus Conference on Definitions of Infection [11]. For example, the diagnosis of community-acquired pneumonia was mainly based on a new infiltrate plus at least one recently acquired respiratory symptom (cough, sputum production, dyspnea, tachypnea, pleuritic pain) or sign (auscultatory findings of abnormal breath sounds and rales). Intraabdominal infections comprised the following diseases in our study: the diagnosis of peritonitis was based on clinical findings including abdominal pain, tenderness to palpation, and peritoneal signs such as rigidity or rebound tenderness, which were supported by radiographic findings, such as free air under the diaphragm or localized fluid collection visualized by computed tomography with a compatible clinical illness; the diagnosis of biliary tract infection was on the basis of clinical evidence of biliary tract infection with surgical or radiographic evidence of supportive complications; typhlitis was diagnosed according to a compatible clinical presentation with radiographic evidence of bowel wall edema and/or gas and/or hemorrhagic necrosis within the bowel wall of the cecum; and the diagnosis of pyelonephritis was based on clinical features (fever $\left(>38^{\circ} \mathrm{C}\right)$, urgency, localized pain or tenderness at involved site), and pyuria, hematuria, and radiographic evidence of infection. Skin and soft-tissue infections (cellulitis) were suggested by the presence of a rapidly expanding erythema, local tenderness, pain, swelling, lymphangitis, and lymphadenopathy, which is frequently accompanied by systemic signs and symptoms including malaise, fever (temperature $>38.0^{\circ} \mathrm{C}$ ), and chills. Determination of bacterial meningitis was based on compatible clinical features, and cerebrospinal fluid findings (cerebrospinal fluid leukocyte count $>1,000 / \mathrm{mm}^{3}$ ) [12] .

\section{Data collection}

Subject data including name, age, sex, past medical history and vital signs were recorded at enrollment. Laboratory examinations, including whole blood leukocyte counts, blood gas analysis, blood biochemistry, X-ray scans and others, were carried out within 24 hours.

Venous blood samples were obtained at ED admission and collected in tubes containing heparin or ethylenediamine tetraacetate and stored at $-80^{\circ} \mathrm{C}$ after collection for analysis within 24 hours. Plasma presepsin concentrations were determined with a compact automated immunoanalyzer (PATHFAST; Mitsubishi Chemical Medience Corporation, Tokyo, Japan) based on a chemiluminescent enzyme immunoassay $[5,6,13]$, and the assay results were obtained within 17 minutes. This assay has a normal reference range of 60 to $365 \mathrm{pg} / \mathrm{ml}$.

PCT was measured by a BioMerieux Mini VIDAS immunoassay analyzer (Block Scientific, Bohemia, NY, USA) in serum samples. Serum was separated by centrifugation at 3,000 rpm for 5 minutes and analyzed within 24 hours. The upper and lower detection limits were $200.0 \mathrm{ng} / \mathrm{ml}$ and $0.05 \mathrm{ng} / \mathrm{ml}$, respectively.

The MEDS score and APACHE II score were calculated according to age, past medical history, vital signs and laboratory results $[14,15]$ when the patients were admitted to the ED.

Septic patients were classified into surviving and nonsurviving groups according to 28 -day survival. The enrolled septic patients who died from all causes within the follow-up time were considered nonsurvivors.

\section{Statistical analysis}

All data were analyzed by SPSS 16.0 software (SPSS Inc., Chicago, IL, USA). Plasma presepsin levels, serum PCT levels, MEDS score and APACHE II score had skewed distributions and were expressed as the median (25th to 75th percentile). For multi-group comparisons, KruskalWallis one-way analysis of variance was applied, and 
two-group comparisons were performed nonparametrically using the Mann-Whitney $\mathrm{U}$ test. To compare the predictive value of presepsin, PCT, MEDS score and APACHE II score for severe sepsis, septic shock and 28-day mortality, receiver operating characteristic (ROC) curves were constructed and the areas under the ROC curves (AUCs) were determined. The outcome variable was 28-day mortality. On the basis of optimal thresholds determined according to ROC curve analysis, prognostic parameters (sensitivity, specificity, positive predictive value (PPV), negative predictive value (NPV), positive likelihood ratio $(\mathrm{LR}+)$ and negative likelihood ratio (LR-)) were also calculated. For comparisons of AUCs, the $Z$-test formula was applied:

$$
Z=(\mathrm{A} 1-\mathrm{A} 2) / \sqrt{\mathrm{SE}_{1}^{2}+\mathrm{SE}_{2}{ }^{2}}
$$

The test standard was $Z_{0.05}=1.96, Z_{0.01}=2.58$. Binary logistic regression analysis was applied to determine the independent predictors of severe sepsis, septic shock, and 28-day mortality. All statistical tests were two-tailed, and $P<0.05$ was considered statistically significant. Spearman correlation analysis was applied to determine the correlation between presepsin, PCT, MEDS score and APACHE II score.

\section{Results}

\section{Characteristics of enrolled subjects}

Between December 2011 and October 2012, 859 patients in the ED of Beijing Chao-yang Hospital and 100 healthy controls were enrolled in this study. No significant differences were found in age, sex and correlative diseases among the five groups (SIRS, sepsis, severe sepsis, septic shock, and control groups). The characteristics, diseases and associated infections in enrolled subjects are presented in Table 1.

\section{Comparison of median levels of presepsin, procalcitonin, MEDS score and APACHE II score}

The median presepsin levels, PCT levels, MEDS score and APACHE II score in each group are shown in Table 1. Plasma presepsin, serum PCT, MEDS score and APACHE II score at ED admission were significantly different among these groups. Compared with the healthy control group, presepsin and PCT levels were significantly higher in patients with SIRS, sepsis, severe sepsis and septic shock $(P<0.0001)$, and presepsin levels, PCT levels and

Table 1 Patient characteristics

\begin{tabular}{|c|c|c|c|c|c|c|}
\hline & Control & SIRS & Sepsis & Severe sepsis & Septic shock & $P$ value \\
\hline$n$ & 100 & 179 & 372 & 210 & 98 & \\
\hline \multirow[t]{2}{*}{ Age (years) } & 68 & 70 & 71 & 73 & 73 & \multirow{2}{*}{0.1} \\
\hline & (65 to 74$)$ & (58 to 76$)$ & (59 to 78 ) & (60 to 78$)$ & (65 to 78$)$ & \\
\hline Male (\%) & 57.0 & 53.6 & 61.2 & 62.9 & 58.2 & 0.5 \\
\hline \multirow[t]{2}{*}{ Presepsin $(\mathrm{pg} / \mathrm{ml})$} & 130 & 212 & 325 & 787 & 1084 & \multirow{2}{*}{0.00} \\
\hline & (104 to 179 ) & (143 to 300$)$ & (210 to 480$)$ & (464 to 1249 ) & (695 to 2365 ) & \\
\hline \multirow[t]{2}{*}{ PCT (ng/ml) } & 0.05 & 0.05 & 0.17 & 1.09 & 6.99 & \multirow{2}{*}{0.00} \\
\hline & (0.05 to 0.07 ) & (0.05 to 0.23 ) & (0.05 to 0.79 ) & (0.21 to 7.52$)$ & (0.88 to 38.26$)$ & \\
\hline \multirow[t]{2}{*}{ MEDS score } & & 6.5 & 8.0 & 14.0 & 19.0 & \multirow{2}{*}{0.00} \\
\hline & & (3.0 to 10.0 ) & (7.0 to 10.0$)$ & (10.0 to 16.0$)$ & (16.8 to 22.0 ) & \\
\hline \multirow[t]{2}{*}{ APACHE II score } & & 13.0 & 14.0 & 19.0 & 22.5 & \\
\hline & & (8.0 to 19.0 ) & (10.0 to 18.0$)$ & (14.0 to 22.0$)$ & (19.0 to 30.25 ) & 0.00 \\
\hline \multicolumn{7}{|l|}{ Diagnosis } \\
\hline \multirow[t]{3}{*}{ Respiratory } & & AECOPD (70) & \multirow[t]{3}{*}{ Pneumonia (259) } & \multirow[t]{3}{*}{ Pneumonia (145) } & \multirow[t]{3}{*}{ Pneumonia (70) } & \multirow[t]{3}{*}{0.92} \\
\hline & & Asthma (38) & & & & \\
\hline & & PE (12) & & & & \\
\hline Abdominal & & Pancreatitis (34) & $|A|(82)$ & $|A|(52)$ & $|A|(22)$ & 0.42 \\
\hline Cerebral & & Stroke (12) & Meningitis (12) & Meningitis (6) & Meningitis (4) & 0.4 \\
\hline Urinary & & & Pyelonephritis (15) & Pyelonephritis (7) & Pyelonephritis (2) & 0.9 \\
\hline Others & & DKA (13) & Skin/soft tissue infection (4) & & & \\
\hline
\end{tabular}

Data are presented as median (25th to 75th percentile). AECOPD, acute exacerbation of chronic obstructive pulmonary diseases; APACHE, Acute Physiology and Chronic Health Evaluation; DKA, diabetic ketoacidosis; IAl, intraabdominal infection; MEDS, Mortality in Emergency Department Sepsis; PE, pulmonary embolism; PCT, procalcitonin; SIRS, systemic inflammatory response syndrome. 
MEDS score were significantly higher in sepsis, severe sepsis and septic shock than in SIRS $(P<0.0001)$. The APACHE II score was significantly higher in severe sepsis and septic shock than in SIRS $(P<0.0001)$, but there was no difference for APACHE II score in sepsis than in SIRS $(P=0.787)$. Presepsin levels, PCT levels, MEDS score and APACHE II score were markedly higher in severe sepsis and septic shock than in sepsis $(P<0.0001)$, and were obviously higher in septic shock than in severe sepsis $(P<0.0001)$. Median presepsin levels, PCT levels, MEDS score and APACHE II score among these groups are illustrated in Figures 1, 2, 3 and 4.

Value of presepsin and procalcitonin for diagnosing sepsis The ROC curves of presepsin and PCT for diagnosing sepsis among the five groups are shown in Figure 5. The AUC of presepsin was 0.820 , significantly higher than that of PCT $(0.724 ; P<0.01)$.

Using a presepsin cutoff value of $317 \mathrm{pg} / \mathrm{ml}$ for diagnosing sepsis, the sensitivity was $70.8 \%$, the specificity was $85.8 \%$, the PPV was $93.2 \%$, the NPV was $51.6 \%$, the predictive accuracy was $74.8 \%$, the $L R+$ was 4.99 , and the LR- was 0.34 .

Using a PCT cutoff value of $0.25 \mathrm{ng} / \mathrm{ml}$ for diagnosing sepsis, the sensitivity was $60.0 \%$, the specificity was $77.7 \%$, the PPV was $92.9 \%$, the NPV was $28.4 \%$, the predictive accuracy was $63.0 \%$, the $\mathrm{LR}+$ was 2.69 , and the LR- was 0.51 .
Value of presepsin, procalcitonin, MEDS score and APACHE II score for predicting severe sepsis

The ROC curves of presepsin, PCT, MEDS score and APACHE II score for predicting severe sepsis in septic patients are displayed in Figure 6. The AUC of presepsin was 0.840 , significantly higher than that of PCT (0.741; $P<0.01)$. The AUC of a combination of presepsin and MEDS score or APACHE II score was significantly higher than that of MEDS score or APACHE II score alone in predicting severe sepsis $(0.875$ vs. 0.818 or 0.858 vs. 0.744 ; all $P<0.01$ ), and there was no difference for the combination of presepsin and MEDS score or APACHE II score compared with presepsin alone (all $P>0.05$ ).

Using a presepsin cutoff value of $449 \mathrm{pg} / \mathrm{ml}$ for predicting severe sepsis, the sensitivity was $82.4 \%$, the specificity was $72.4 \%$, the PPV was $71.3 \%$, the NPV was $83.2 \%$, the predictive accuracy was $77.0 \%$, the LR + was 2.99 , and the LR- was 0.24 . The detailed results are displayed in Tables 2 and 3.

\section{Value of presepsin, procalcitonin, MEDS score and APACHE II score for predicting septic shock}

The AUC of presepsin for predicting septic shock was 0.790, higher than that of PCT (0.768), but was not statistically significant $(P>0.05)$. The AUC of a combination of presepsin and MEDS score or APACHE II score was significantly higher than that of presepsin alone in predicting septic shock (0.924 vs. $0.790, P<0.01 ; 0.868$ vs. 0.790 ,

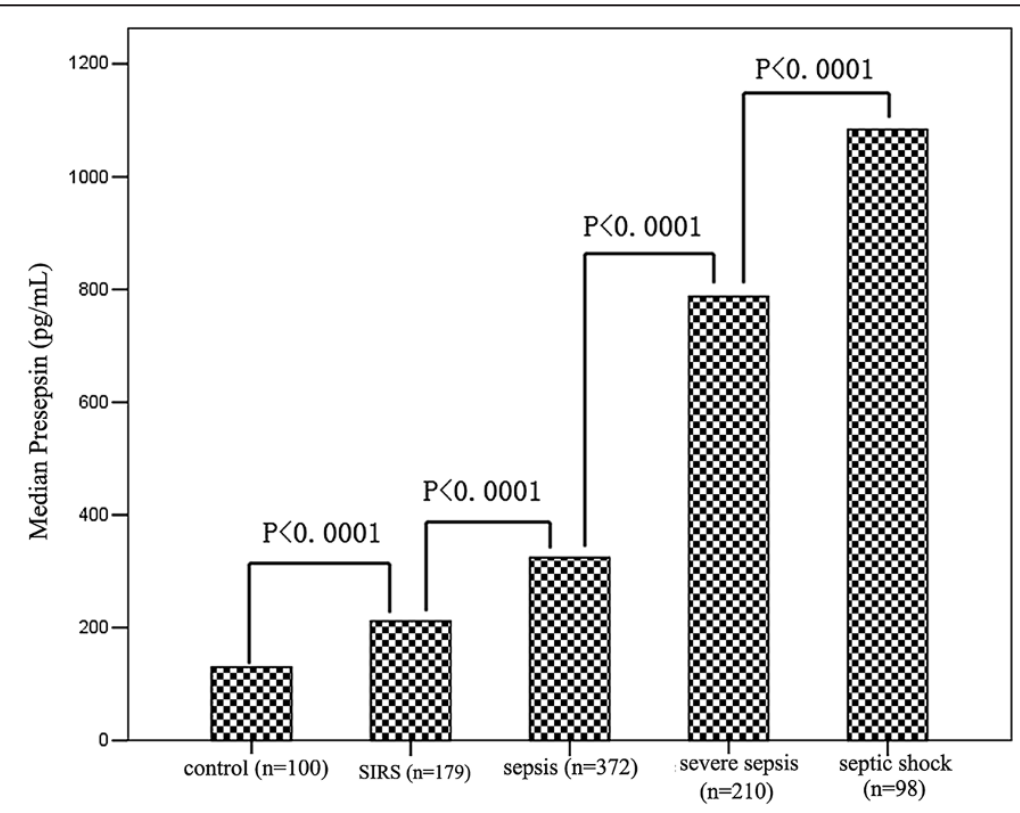

Figure 1 Plasma presepsin levels in healthy control individuals, and patients with SIRS, sepsis, severe sepsis and septic shock at emergency department admission. Columns represent median presepsin levels. Numbers of samples are indicated in parentheses. SIRS, systemic inflammatory response syndrome. 


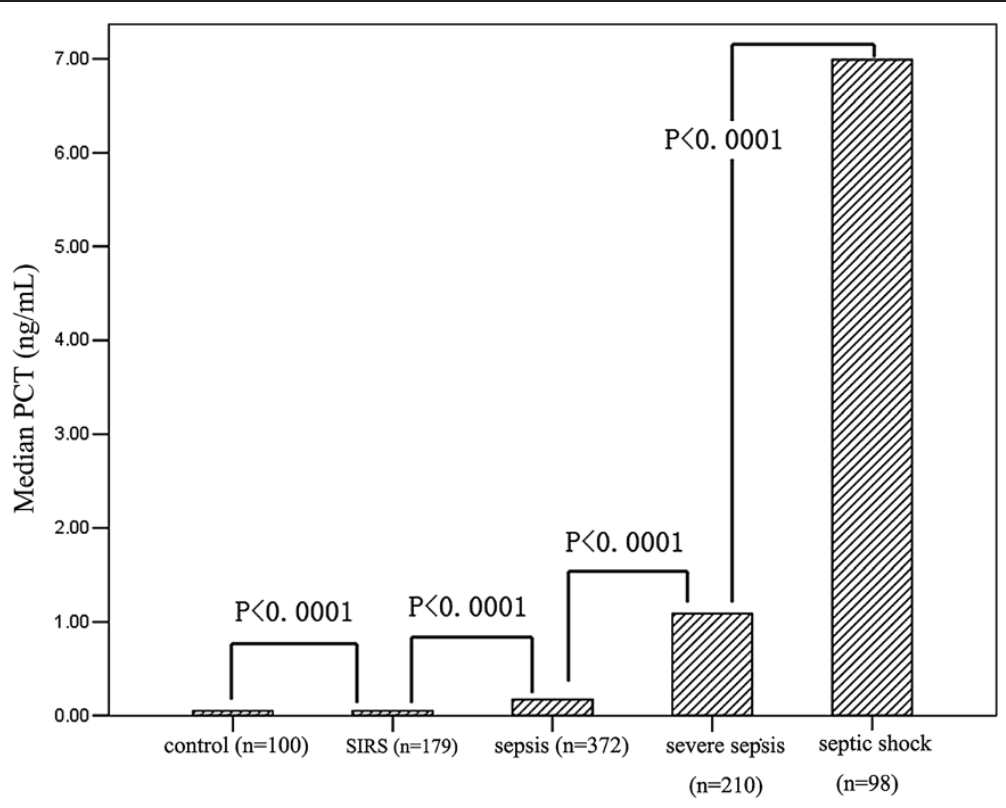

Figure 2 Serum procalcitonin levels in healthy control individuals, and patients with SIRS, sepsis, severe sepsis and septic shock at emergency department admission. Columns represent median procalcitonin (PCT) levels. Numbers of samples are indicated in parentheses. SIRS, systemic inflammatory response syndrome.

$P<0.05)$, and there was no difference for the combination of presepsin and MEDS score or APACHE II score compared with MEDS score or APACHE II score alone (all $P>0.05)$, respectively. The AUC of a combination of presepsin and MEDS score was greater than that of a combination of presepsin and APACHE II score $(P<0.05)$.
Using a presepsin cutoff value of $550 \mathrm{pg} / \mathrm{ml}$ for predicting septic shock, the sensitivity was $85.7 \%$, the specificity was $63.6 \%$, the PPV was $28.5 \%$, the NPV was $96.3 \%$, the predictive accuracy was $66.8 \%$, the LR + was 2.35 , and the LR- was 0.22 . The detailed results are demonstrated in Tables 2 and 3.

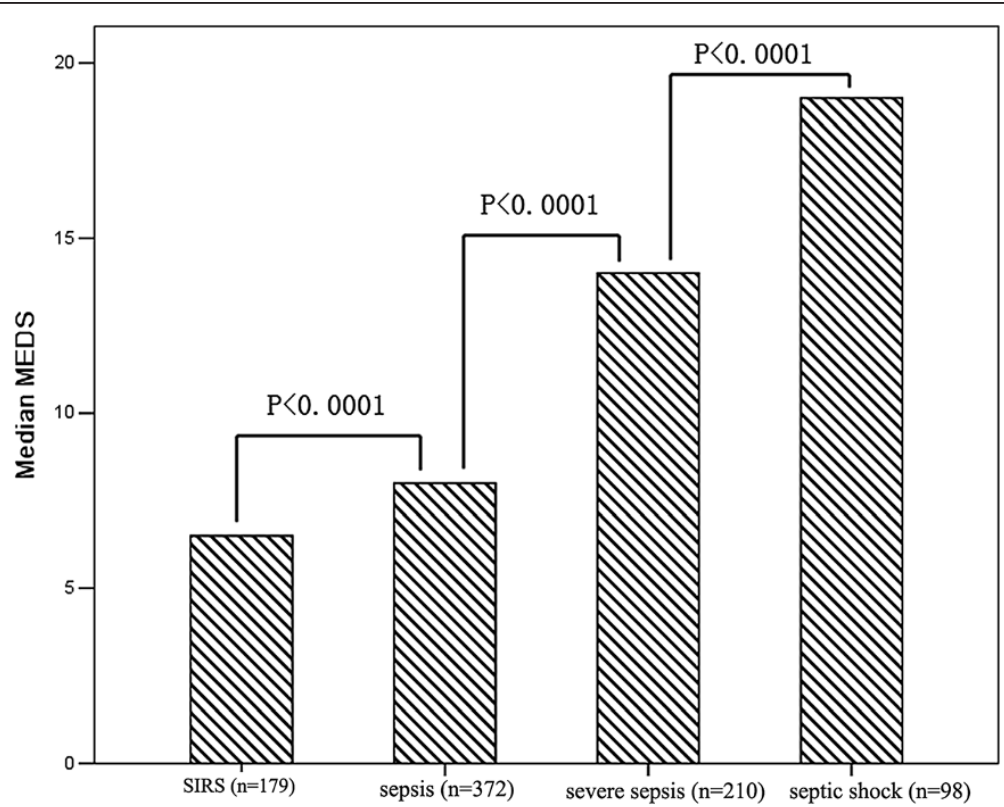

Figure 3 MEDS score in patients with SIRS, sepsis, severe sepsis and septic shock at emergency department admission. Columns represent median Mortality in Emergency Department Sepsis (MEDS) score levels. Numbers of samples are indicated in parentheses. SIRS, systemic inflammatory response syndrome. 


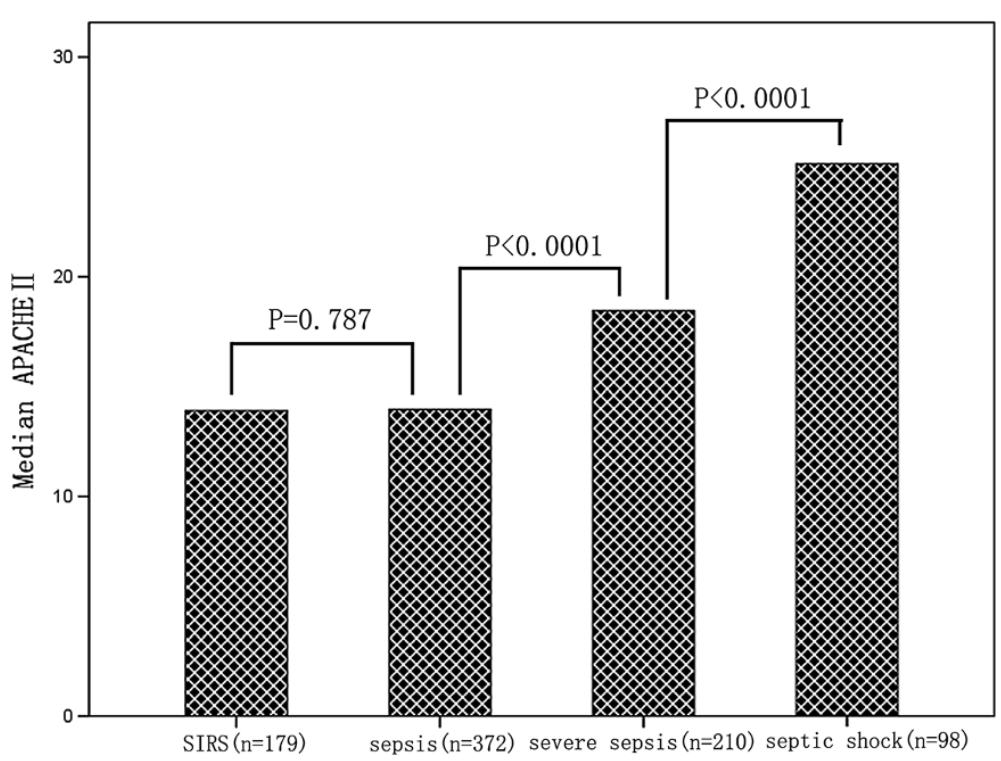

Figure 4 APACHE II score in patients with SIRS, sepsis, severe sepsis and septic shock at emergency department admission. Columns represent median Acute Physiology and Chronic Health Evaluation (APACHE) II score levels. Numbers of samples are indicated in parentheses. SIRS, systemic inflammatory response syndrome.

Value of presepsin, procalcitonin, MEDS score and APACHE II score for predicting 28-day mortality The AUC of presepsin for predicting 28-day mortality in septic patients was 0.658 , slightly lower than that of PCT $(0.679 ; P>0.05)$, MEDS score $(0.719 ; P>0.05)$, and

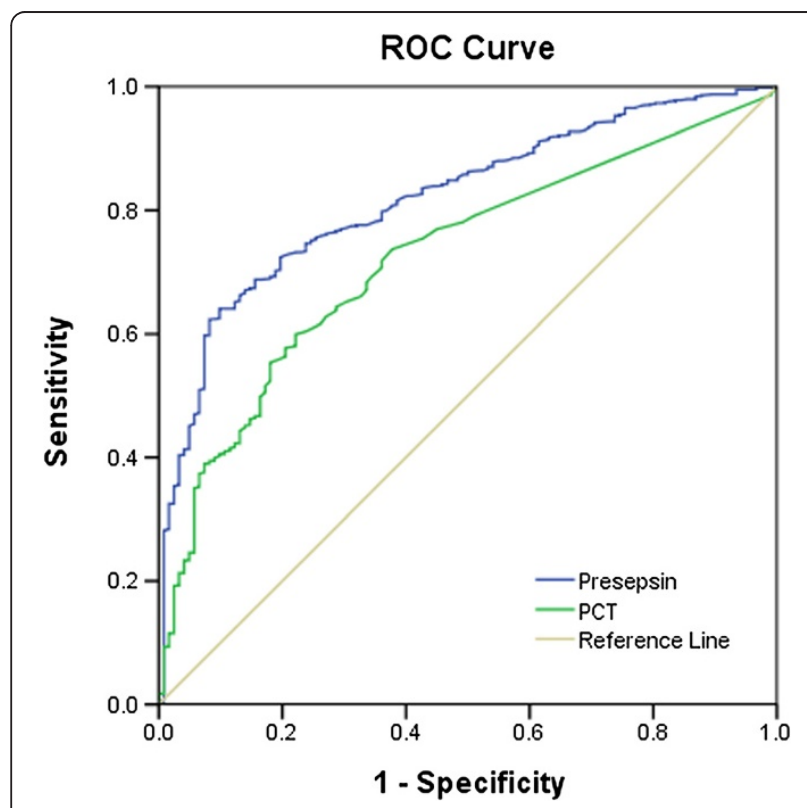

Figure 5 Receiver operating characteristic curves of presepsin and procalcitonin for diagnosis of sepsis. Areas under the receiver operating characteristic (ROC) curve: presepsin (blue line), 0.820 (95\% confidence interval: 0.784 to 0.856$), P<0.0001$; and procalcitonin (PCT; green line), 0.724 (95\% confidence interval: 0.680 to 0.769$), P<0.0001$.

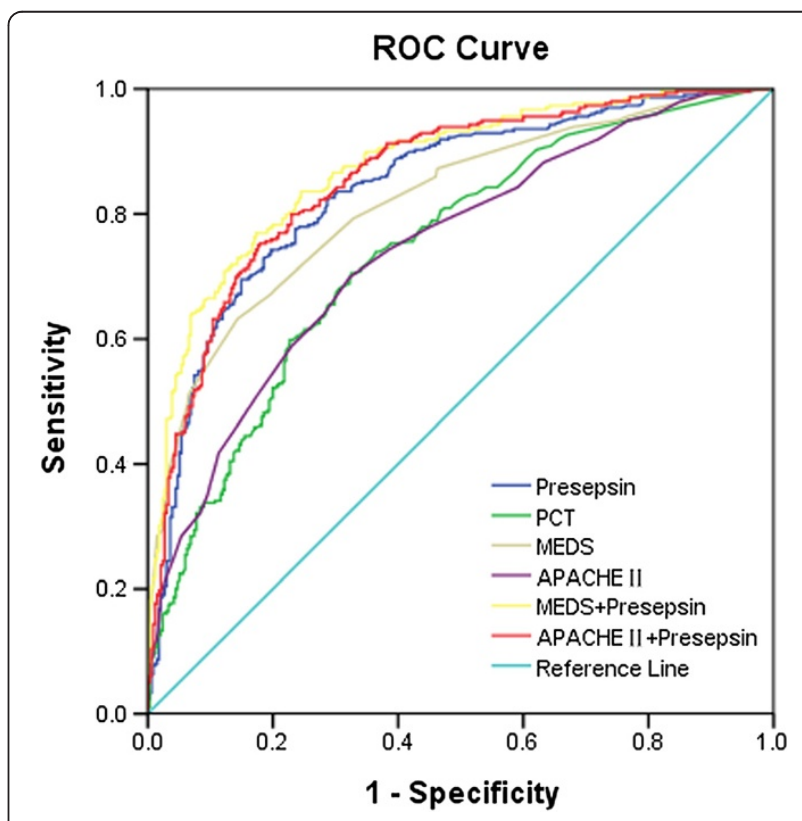

Figure 6 Receiver operating characteristic curves of presepsin, procalcitonin, MEDS score and APACHE II score for predicting severe sepsis in septic patients. Areas under the receiver operating characteristic (ROC) curves: presepsin (blue line), 0.840 ( $95 \%$ confidence interval (Cl): 0.809 to 0.872$), P<0.0001$; procalcitonin (PCT; green line), 0.741 (95\% Cl: 0.703 to 0.779), $P<0.0001$; Mortality in Emergency Department Sepsis (MEDS) score (brown line), 0.818 (95\% Cl: 0.785 to 0.851), $P<0.0001$; Acute Physiology and Chronic Health Evaluation (APACHE) II score (purple line), 0.744 ( $95 \%$ Cl: 0.706 to 0.782), $P<0.0001$; presepsin in combination with MEDS score (yellow line), 0.875 (95\% Cl: 0.848 to 0.901), $P<0.0001$; and presepsin in combination with APACHE II score (pink line), 0.858 (95\% Cl: 0.829 to 0.887 ), $P<0.0001$. 
Table 2 Areas under the curve of various parameters for predicting severe sepsis, septic shock and 28-day mortality in septic patients

\begin{tabular}{|c|c|c|c|c|c|c|}
\hline & \multirow[t]{2}{*}{ Variable } & \multirow[t]{2}{*}{ AUC } & \multirow[t]{2}{*}{ Standard error } & \multirow[t]{2}{*}{$P$ value } & \multicolumn{2}{|c|}{ 95\% confidence interval } \\
\hline & & & & & Lower limit & Upper limit \\
\hline \multirow[t]{6}{*}{ Severe sepsis } & Presepsin & 0.840 & 0.016 & 0 & 0.809 & 0.872 \\
\hline & Procalcitonin & 0.741 & 0.019 & 0 & 0.703 & 0.779 \\
\hline & MEDS score & 0.818 & 0.017 & 0 & 0.785 & 0.851 \\
\hline & APACHE II score & 0.744 & 0.019 & 0 & 0.706 & 0.782 \\
\hline & MEDS score + presepsin ${ }^{a}$ & 0.875 & 0.014 & 0 & 0.848 & 0.901 \\
\hline & APACHE II score + presepsin ${ }^{b}$ & 0.858 & 0.015 & 0 & 0.829 & 0.887 \\
\hline \multirow[t]{6}{*}{ Septic shock } & Presepsin & 0.790 & 0.024 & 0 & 0.742 & 0.837 \\
\hline & Procalcitonin & 0.768 & 0.024 & 0 & 0.721 & 0.816 \\
\hline & MEDS score & 0.904 & 0.017 & 0 & 0.871 & 0.938 \\
\hline & APACHE II score & 0.820 & 0.023 & 0 & 0.774 & 0.865 \\
\hline & MEDS score + presepsin $^{c}$ & 0.924 & 0.014 & 0 & 0.897 & 0.951 \\
\hline & APACHE $\|$ score + presepsin $^{d}$ & 0.868 & 0.019 & 0 & 0.831 & 0.905 \\
\hline \multirow[t]{6}{*}{ 28-day mortality } & Presepsin & 0.658 & 0.023 & 0 & 0.614 & 0.703 \\
\hline & Procalcitonin & 0.679 & 0.022 & 0 & 0.636 & 0.722 \\
\hline & MEDS score & 0.719 & 0.021 & 0 & 0.677 & 0.760 \\
\hline & APACHE I| score & 0.722 & 0.021 & 0 & 0.681 & 0.763 \\
\hline & MEDS score + presepsin ${ }^{e}$ & 0.731 & 0.021 & 0 & 0.690 & 0.771 \\
\hline & APACHE II score + presepsin ${ }^{f}$ & 0.734 & 0.021 & 0 & 0.693 & 0.775 \\
\hline
\end{tabular}

AUC, area under the receiver operating characteristic curve. ${ }^{a}$ Compared with Mortality in Emergency Department Sepsis (MEDS) score, $P<0.01 .{ }^{b}$ Compared with Acute Physiology and Chronic Health Evaluation (APACHE) II score, $P<0.01$. ${ }^{\mathrm{C}}$ Compared with presepsin, $P<0.01$. ${ }^{\mathrm{d}} \mathrm{Compared}$ with presepsin, $P<0.05$. ${ }^{\mathrm{C}} \mathrm{Compared}$ with presepsin, $P<0.05$. ${ }^{\text {f }}$ Compared with presepsin, $\mathrm{P}<0.05$.

APACHE II score $(0.722 ; P<0.05)$. The AUC of presepsin in combination with MEDS score or APACHE II score was 0.731 or 0.734 , which was more statistically significant compared with presepsin alone (0.658; all $P<0.05)$, and there was no difference for the combination of presepsin and MEDS score or APACHE II score compared with MEDS score or APACHE II score alone (all $P>0.05)$, respectively.

Using a presepsin cutoff value of $556 \mathrm{pg} / \mathrm{ml}$ for predicting 28-day mortality in septic patients, the sensitivity

Table 3 Performance of multivariable models for predicting severe sepsis, septic shock and 28-day mortality in septic patients

\begin{tabular}{|c|c|c|c|c|c|c|c|c|c|}
\hline & Variable & Cutoff & Sensitivity (\%) & Specificity (\%) & PPV (\%) & NPV (\%) & Accuracy (\%) & LR+ & $\overline{\mathrm{LR}-}$ \\
\hline \multirow[t]{4}{*}{ Severe sepsis } & Presepsin & $449 \mathrm{pg} / \mathrm{ml}$ & 82.4 & 72.4 & 71.3 & 83.2 & 77.0 & 2.99 & 0.24 \\
\hline & Procalcitonin & $1.435 \mathrm{ng} / \mathrm{ml}$ & 52.0 & 79.8 & 69.6 & 65.1 & 66.7 & 2.57 & 0.60 \\
\hline & MEDS score & 13.5 score & 63.0 & 86.3 & 79.2 & 73.8 & 75.8 & 4.60 & 0.42 \\
\hline & APACHE II score & 16.5 score & 69.8 & 68.5 & 64.8 & 73.3 & 69.1 & 2.22 & 0.44 \\
\hline \multirow[t]{4}{*}{ Septic shock } & Presepsin & $550 \mathrm{pg} / \mathrm{ml}$ & 85.7 & 63.6 & 28.5 & 96.3 & 66.8 & 2.35 & 0.22 \\
\hline & Procalcitonin & $4.415 \mathrm{ng} / \mathrm{ml}$ & 54.1 & 81.1 & 34.2 & 90.7 & 77.0 & 2.86 & 0.57 \\
\hline & MEDS score & 16.5 score & 75.5 & 90.0 & 56.1 & 95.6 & 87.9 & 7.55 & 0.27 \\
\hline & APACHE II score & 20.5 score & 70.4 & 78.4 & 35.4 & 94.0 & 77.2 & 3.26 & 0.38 \\
\hline \multirow[t]{4}{*}{ 28-day mortality } & Presepsin & $556 \mathrm{pg} / \mathrm{ml}$ & 62.2 & 66.8 & 48.3 & 78.0 & 65.3 & 1.87 & 0.57 \\
\hline & Procalcitonin & $1.125 \mathrm{ng} / \mathrm{ml}$ & 54.2 & 70.0 & 47.7 & 75.1 & 64.7 & 1.81 & 0.65 \\
\hline & MEDS score & 13.5 score & 59.1 & 75.4 & 54.3 & 78.9 & 70.0 & 2.40 & 0.54 \\
\hline & APACHE II score & 16.5 score & 71.1 & 62.2 & 48.2 & 81.3 & 65.1 & 1.88 & 0.46 \\
\hline
\end{tabular}


was $62.2 \%$, the specificity was $66.8 \%$, the PPV was $48.3 \%$, the NPV was $78.0 \%$, the predictive accuracy was $65.3 \%$, the LR + was 1.87, and the LR- was 0.57. The detailed results are presented in Tables 2 and 3.

Comparison of median levels of presepsin at admission in nonsurviving and surviving groups of septic patients at 28-day follow-up

The median levels of presepsin were significantly higher in nonsurvivors than in survivors $(748 \mathrm{pg} / \mathrm{ml}$ (385 to $1386 \mathrm{pg} / \mathrm{ml}$ ) vs. $412 \mathrm{pg} / \mathrm{ml}$ (243 to $744 \mathrm{pg} / \mathrm{ml}) ; P<0.0001)$. The detailed results are illustrated in Figure 7.

Presepsin, procalcitonin, MEDS score and APACHE II score as independent predictors of severe sepsis, septic shock and 28-day mortality

Using binary logistic regression analysis, presepsin $(B=$ 0.001 , odds ratio $(\mathrm{OR})=1.001, P=0)$, MEDS score $(B=$ 0.232 , $\mathrm{OR}=1.262, P=0)$ and APACHE II score $(B=0.081$, $\mathrm{OR}=1.085, P=0$ ) were found to be the independent predictors of severe sepsis in septic patients, but PCT $(B=$ 0.010, $\mathrm{OR}=1.010, P=0.069)$ was not (Table 4$)$.

Presepsin $(B=0.000, \mathrm{OR}=1.000, P=0)$, PCT $(\mathrm{B}=0.008$, $\mathrm{OR}=1.008, P=0.025)$, MEDS score $(B=0.347$, OR $=$ $1.415, P=0)$ and APACHE II score $(B=0.094$, OR $=1.098$, $P=0$ ) were found to be the independent predictors of septic shock in septic patients (Table 4).

Presepsin $(B=0.000, \quad \mathrm{OR}=1.000, P=0.021)$, MEDS score $(B=0.088, \mathrm{OR}=1.091, P=0)$ and APACHE II score $(B=0.080$, OR $=1.083, P=0)$ were found to be independent predictors of 28-day mortality in septic patients, but PCT $(B=0.002, \mathrm{OR}=1.002, P=0.536)$ was not (Table 4).

\section{Correlation of plasma presepsin levels with serum} procalcitonin levels, MEDS score and APACHE II score in septic patients

Spearman correlation analysis of presepsin with PCT, MEDS score and APACHE II score showed that, compared with PCT, MEDS score and APACHE II score, the correlation coefficients of presepsin were 0.502, 0.414 and 0.294 (all $P<0.0001$ ), respectively, which suggested significantly positive correlations.

\section{Discussion}

Despite the use of antimicrobial agents and advanced life support, the case fatality rate for patients with sepsis has remained between 20 and 30\% during the past two decades $[16,17]$.

Biomarkers play a pivotal pole in early diagnosis, differential diagnosis, risk stratification, therapy monitoring and evaluation of prognosis of sepsis. Presepsin, generated by circulating plasma proteases activating cleavage of soluble CD14 [18], is a novel biomarker that has been used for diagnosis of sepsis in recent years, and its production is associated with phagocytosis and cleavage of microorganisms by lysosomal enzymes [19].

In this study, we found that presepsin levels increased in early sepsis, and that the levels were significantly higher than in healthy controls and in SIRS patients, which was in accordance with previous results $[4,5,9]$. In addition, with progression of sepsis, the plasma presepsin levels

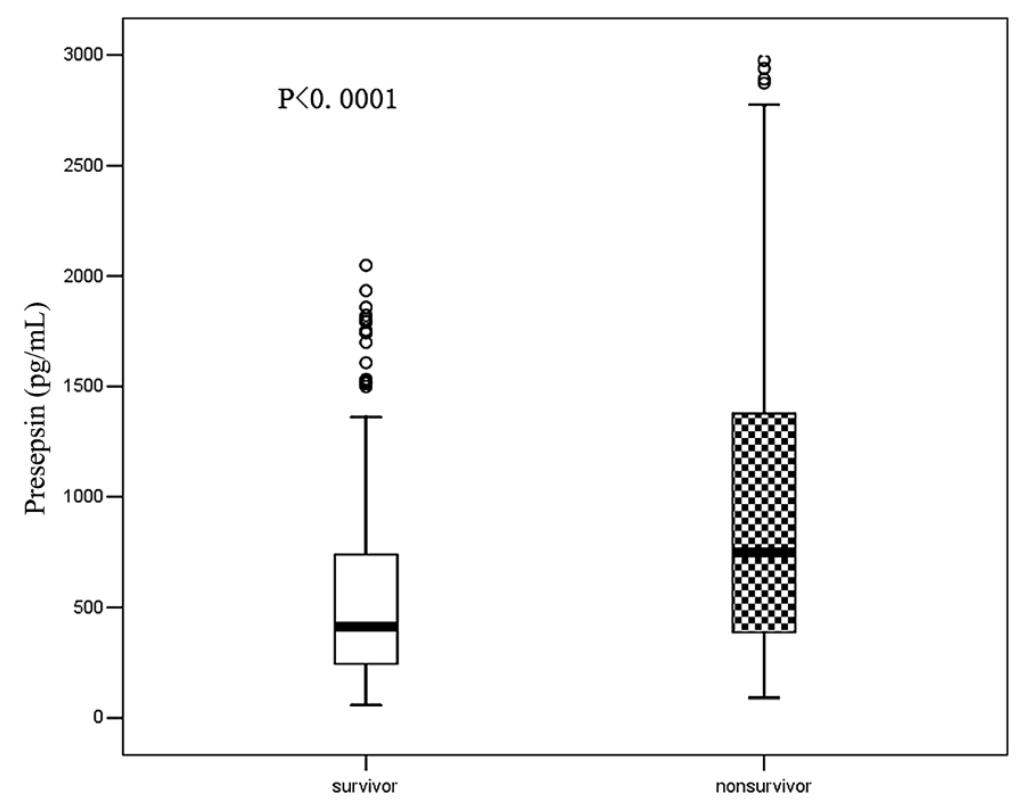

Figure 7 Presepsin levels at admission in surviving and nonsurviving groups of septic patients at 28-day follow-up. Lines denote median values, boxes represent 25 th to 75 th percentiles and whiskers indicate the range. 
Table 4 Independent factors predicting severe sepsis, septic shock and 28-day mortality in septic patients

\begin{tabular}{|c|c|c|c|c|c|c|c|c|c|}
\hline & \multirow[t]{2}{*}{ Variable } & \multirow[t]{2}{*}{ B } & \multirow[t]{2}{*}{ Standard error } & \multirow[t]{2}{*}{ Wald } & \multirow[t]{2}{*}{ Degrees of freedom } & \multirow[t]{2}{*}{$P$ value } & \multirow[t]{2}{*}{ Odds ratio } & \multicolumn{2}{|c|}{$95 \%$ confidence interval } \\
\hline & & & & & & & & Lower limit & Upper limit \\
\hline \multirow[t]{7}{*}{ Severe sepsis } & Presepsin & 0.001 & 0.000 & 39.938 & 1 & 0.000 & 1.001 & 1.001 & 1.002 \\
\hline & Procalcitonin & 0.010 & 0.005 & 3.298 & 1 & 0.069 & 1.010 & 0.999 & 1.021 \\
\hline & MEDS score & 0.232 & 0.029 & 63.948 & 1 & 0.000 & 1.262 & 1.192 & 1.336 \\
\hline & APACHE II score & 0.081 & 0.019 & 17.804 & 1 & 0.000 & 1.085 & 1.044 & 1.126 \\
\hline & Age & -0.028 & 0.009 & 9.607 & 1 & 0.002 & 0.973 & 0.956 & 0.990 \\
\hline & Gender & -1.101 & 0.224 & 0.204 & 1 & 0.651 & 0.904 & 0.582 & 1.403 \\
\hline & Constant & -3.223 & 0.697 & 21.374 & 1 & 0.000 & 0.040 & & \\
\hline \multirow[t]{7}{*}{ Septic shock } & Presepsin & 0.000 & 0.000 & 13.308 & 1 & 0.000 & 1.000 & 1.000 & 1.001 \\
\hline & Procalcitonin & 0.008 & 0.004 & 5.014 & 1 & 0.025 & 1.008 & 1.001 & 1.025 \\
\hline & MEDS score & 0.347 & 0.045 & 58.391 & 1 & 0.000 & 1.415 & 1.295 & 1.547 \\
\hline & APACHE II score & 0.094 & 0.024 & 14.928 & 1 & 0.000 & 1.098 & 1.047 & 1.151 \\
\hline & Age & -0.027 & 0.012 & 5.285 & 1 & 0.022 & 0.973 & 0.951 & 0.996 \\
\hline & Gender & -0.081 & 0.325 & 0.062 & 1 & 0.803 & 0.922 & 0.487 & 1.744 \\
\hline & Constant & -7.462 & 1.083 & 47.511 & 1 & 0.000 & 0.001 & & \\
\hline \multirow[t]{7}{*}{ 28-day mortality } & Presepsin & 0.000 & 0.000 & 5.296 & 1 & 0.021 & 1.000 & 1.000 & 1.000 \\
\hline & Procalcitonin & 0.002 & 0.003 & 0.382 & 1 & 0.536 & 1.002 & 0.996 & 1.008 \\
\hline & MEDS score & 0.088 & 0.022 & 15.223 & 1 & 0.000 & 1.091 & 1.045 & 1.141 \\
\hline & APACHE II score & 0.080 & 0.016 & 25.658 & 1 & 0.000 & 1.083 & 1.050 & 1.117 \\
\hline & Age & 0.004 & 0.007 & 0.346 & 1 & 0.556 & 1.004 & 0.990 & 1.019 \\
\hline & Gender & -0.038 & 0.194 & 0.038 & 1 & 0.846 & 0.963 & 0.658 & 1.409 \\
\hline & Constant & -3.651 & 0.623 & 34.307 & 1 & 0.000 & 0.026 & & \\
\hline
\end{tabular}

APACHE, Acute Physiology and Chronic Health Evaluation; MEDS, Mortality in Emergency Department Sepsis.

increased accordingly, and reached the highest levels in septic shock. The present study demonstrated that plasma presepsin levels were a good parameter for reflecting the severity of sepsis.

More recently, PCT has also been used widely as a biomarker for sepsis diagnosis, risk stratification, evaluation of prognosis, and therapy monitoring. Most studies that have examined the discriminative ability of serum PCT in the diagnosis of sepsis have been small, with heterogeneity in the patients enrolled, and have used different cutoff values, and hence have provided variable results [20]. In this study, the cutoff value of PCT for diagnosing sepsis according to the ROC curve was $0.25 \mathrm{ng} / \mathrm{ml}$, lower than that $(0.5 \mathrm{ng} / \mathrm{ml})$ commonly used in clinical settings $[21,22]$. Possible reasons for the difference were as follows: first, the SIRS and septic patients were enrolled according to ACCP/SCCM criteria, not PCT, which was only considered as a comparative index; second, the site of infection in the majority of enrolled patients was located in the lower respiratory tract, thus the PCT cutoff level of $0.25 \mathrm{ng} / \mathrm{ml}$ was usually used for diagnosing low respiratory tract infection [23,24]; third, the septic patients were enrolled and blood samples were obtained at the time of ED admission, when the concentration of PCT had not increased fully or reached a maximum; and fourth, the septic patients may have been treated with antimicrobial agents outside hospital, which may affect the results for PCT, as well as those for presepsin. Furthermore, the median levels of PCT in the present study were lower than the commonly used cutoff values in different clinical settings (Table 1), and the cutoff level of presepsin $(317 \mathrm{pg} / \mathrm{ml})$ was lower than the previously reported cutoff values of 400 to $600 \mathrm{pg} / \mathrm{ml}$ for diagnosing sepsis $[5,9]$.

Although serum PCT can be used as a biomarker in the diagnosis of sepsis, PCT is also increased in other conditions, such as multiple trauma, extensive burns, pancreatitis, organ transplantation, major surgery, and SIRS, but not in infection [25], and its PPV and NPV values are not sufficient to rule in or rule out sepsis in a standalone test. A recent meta-analysis found that the diagnostic performance of PCT was low, with 71\% (95\% confidence interval 67 to $76 \%$ ) sensitivity and specificity for serum PCT as a biomarker of sepsis. PCT therefore cannot reliably differentiate sepsis from other conditions in critically ill adult patients [26].

Compared with PCT, presepsin is a highly specific biomarker for diagnosing bacterial infections because it is produced in association with bacterial phagocytosis. 
Presepsin was shown to be secreted from granulocytes by infectious stimuli in an animal sepsis model [19]. In this study, ROC analysis of a large sample of septic patients at ED admission demonstrated that presepsin was superior to PCT and showed higher sensitivity, specificity, PPV, NPV and predictive accuracy in the early diagnosis of sepsis, which was in line with previous reports $[4,5,9]$.

There was higher mortality as the severity of sepsis increased, and more than 50\% mortality in severe sepsis [17]. Dellinger and colleagues, in the Early-Goal Directed Therapy in 2013 Guidelines of the Surviving Sepsis Campaign [27], recommended that a potential infection source should be confirmed as promptly as possible within the first 6 hours of presentation and that broad-spectrum antibiotic treatment must be administered within 1 hour after the recognition of severe sepsis and septic shock. Early identification of these high-risk patients is therefore crucial. In this study, ROC analysis showed that the AUC of presepsin (0.840) was markedly higher than that of PCT (0.741) $(P<0.01)$ and displayed higher sensitivity $(82.4 \%)$ and NPV (83.2\%) in predicting severe sepsis. Meanwhile, in logistic regression analysis, presepsin was found to be an independent predictor of severe sepsis, but PCT was not, which further indicated that presepsin was superior to PCT in predicting severe sepsis.

Meanwhile, presepsin demonstrated high sensitivity (85.7\%) and high NPV (96.3\%) in predicting septic shock, and plasma presepsin levels lower than the above-mentioned cutoff value $(550 \mathrm{pg} / \mathrm{ml})$ may help rule out the possibility of septic shock. Additionally, in the logistic regression analysis, presepsin and PCT were found to be independent predictors of septic shock in septic patients, which indicated presepsin and PCT had certain value in predicting septic shock.

Scoring systems are also widely applied to risk stratification and prediction of prognosis in septic patients. In the present study, we found that MEDS score and APACHE II score increased along with progression of sepsis, reaching a maximum in septic shock. Similarly, MEDS score and APACHE II score were also a good parameter to reflect the severity of sepsis.

In this study, we further found that presepsin, PCT, MEDS score and APACHE II score were statistically significant for predicting severe sepsis, septic shock and 28-day mortality (all $P<0.0001$ ). The AUC of presepsin in combination with MEDS score or APACHE II score was significantly higher than MEDS score or APACHE II score alone in predicting severe sepsis $(P<0.01)$, and although there was no difference compared with presepsin alone (all $P>0.05$ ), presepsin in combination with MEDS score or APACHE II score markedly increased AUC ( 0.875 or 0.858 , respectively), which indicated that the combination of presepsin with MEDS score or APACHE
II score obviously improved the accuracy of predicting severe sepsis.

Meanwhile, we also found the AUC of presepsin in combination with MEDS score or APACHE II score was significantly higher than presepsin alone in predicting septic shock $(P<0.01)$. Although there was no difference compared with MEDS score or APACHE II score alone (all $P>0.05$ ), presepsin in combination with MEDS score or APACHE II score significantly increased the AUC (0.924 or 0.868 , respectively). In addition, the AUC of presepsin in combination with MEDS score was significantly higher than that of presepsin in combination with APACHE II score $(P<0.05)$. Taken together, combination presepsin with MEDS score or APACHE II score markedly enhanced the accuracy of predicting septic shock, and the combination of presepsin with MEDS score was superior to the combination of presepsin with APACHE II score in predicting septic shock.

Although the AUC of presepsin was slightly lower than that of PCT and MEDS score in predicting 28-day mortality in septic patients, no significant differences were found. However, the AUC of presepsin in combination with MEDS score or APACHE II score was significantly higher than presepsin alone (all $P<0.01)$. Although the sensitivity (62.2\%), the specificity (66.8\%), the PPV (48.3\%), and the NPV (78\%) were a little low for presepsin for predicting 28-day mortality in septic patients, in the logistic regression analysis presepsin, MEDS score and APACHE II score were found to be independent predictors of 28-day mortality in septic patients, while PCT was not - this is in accordance with previous results from Ruiz-Alvarez and colleagues [28], suggesting presepsin was superior to PCT for predicting 28-day mortality in septic patients, and the combination of presepsin with MEDS score or APACHE II score significantly enhanced the accuracy of predicting 28-day mortality in septic patients.

Additionally, Spearman correlation analysis of presepsin found a significantly positive correlation with MEDS score and APACHE II score, indicating that presepsin, MEDS score and APACHE II score may facilitate evaluation of the severity of sepsis and allow effective risk stratification, as presepsin in combination with MEDS score or APACHE II score further enhanced the accuracy of evaluation of septic patients.

The median levels of presepsin were significantly higher in nonsurvivors than in survivors, which further confirmed that the higher the plasma presepsin level, the more adverse the outcome in septic patients. The plasma presepsin level was also a good index for evaluation of prognosis in septic patients.

\section{Limitations}

Although the present study contained a relatively large sample size, some limitations merit consideration. First, 
it was a single-center study and did not compare with other biomarkers and severity score systems. Second, because it was difficult to obtain pathogen samples in an emergency room setting, blood culture and sputum sample were not taken and tested, and sepsis was established on the basis of clinical features, laboratory findings and imaging tests according to criteria for sepsis as defined by ACCP/SCCM.

\section{Conclusions}

This study indicated that presepsin was a more valuable biomarker than PCT in the early diagnosis of sepsis, and presepsin in combination with MEDS score or APACHE II score significantly increased the prognostic accuracy in septic patients. Presepsin thus appears to be a promising biomarker and is superior to PCT for early diagnosis of sepsis, and risk stratification and evaluation of prognosis in septic patients in the ED.

\section{Key messages}

- Plasma presepsin levels were a good parameter for reflecting the severity of sepsis and evaluating prognosis of septic patients.

- Presepsin was superior to PCT for diagnosing sepsis, and predicting severe sepsis, septic shock and 28-day mortality in septic patients in the ED.

- Presepsin, MEDS score and APACHE II score were all independent predictors of severe sepsis, septic shock and 28-day mortality in septic patients.

- Presepsin in combination MEDS score or APACHE II score markedly enhanced the predictive accuracy of predicting severe sepsis, septic shock and 28-day mortality in septic patients.

\footnotetext{
Abbreviations

ACCP: American college of chest physicians; APACHE: Acute physiology and chronic health evaluation; AUC: Area under the receiver operating characteristic curve; ED: Emergency department; LR-: Negative likelihood ratio; LR+: Positive likelihood ratio; MEDS: Mortality in emergency department sepsis; NPV: Negative predictive value; PCT: Procalcitonin; PPV: Positive predictive value; ROC: Receiver operating characteristic; SCCM: Society of critical care medicine; SIRS: Systemic inflammatory response syndrome.
}

\section{Competing interests}

The authors declare they have no competing interests.

\section{Authors' contributions}

C-SL conceived this study, designed the trial and obtained research funding. $B L, Y-X C, Q Y$ and $Y-Z Z$ conducted the trial and collected data. BL analyzed data, performed statistical analysis, drafted and revised the manuscript. C-SL takes responsibility for the paper as a whole. All authors read and approved the final manuscript.

\section{Acknowledgements}

The authors sincerely thank Zi-Ren Tang and Shuo Wang for their excellent assistance. The authors also thank both the ED staff and Biochemistry Laboratory staff for their helpful contributions.

\section{Funding}

This study was supported by National Clinical Key Specialty Construction Project Funds (No. 2012-649) and 2012 Beijing City Outstanding Doctoral Dissertation Funds (No. 2012-1002501).

Received: 15 April 2013 Accepted: 24 September 2013 Published: 20 October 2013

\section{References}

1. Protti A, Singer M: Bench-to-bedside review: potential strategies to protect or reverse mitochondrial dysfunction in sepsis-induced organ failure. Crit Care 2006, 10:228.

2. Wright SD, Ramos RA, Tobias PS, Ulevitch RJ, Mathison JC: CD14, a receptor for complexes of lipopolysaccharide (LPS) and LPS binding protein. Science 1990, 249:1431-1433.

3. Shirakawa K, Naitou K, Hirose J, Takahashi T, Furusako S: Presepsisn (sCD14ST): development and evaluation of one-step ELISA with a new standard that is similar to the form of presepsin in septic patients. Clin Chem Lab Med 2011, 49:937-939.

4. Yaegashi Y, Shirakawa K, Sato N, Suzuki Y, Kojika M, Imai S, Takahashi G, Miyata M, Furusako S, Endo S: Evaluation of a newly identified soluble CD14 subtype as a marker for sepsis. J Infect Chemother 2005, 11:234-238.

5. Shozushima T, Takahashi G, Matsumoto N, Kojika M, Okamura Y, Endo S: Usefulness of presepsin (sCD14-ST) measurements as a marker for the diagnosis and severity of sepsis that satisfied diagnostic criteria of systemic inflammatory response syndrome. J Infect Chemother 2011, 17:764-769

6. Okamura $Y$, Yokoi H: Development of a point-of-care assay system for measurement of presepsin (sCD14-ST). Clin Chim Acta 2011, 412:2157-2161.

7. Endo S, Yaegashi Y, Sato N, Suzuki Y, Ogawa M, Kojika M: Comparative study of soluble CD14 and soluble CD14-subtype in sepsis. Med Postgrad 2006, 44:381-385.

8. Miyata M, Sato N, Takahashi G, Takahashi M, Endo S: The utility of the soluble CD-14-subtype for diagnosis of sepsis, the examination of the simple diagnostic kit. I Iwate Med Assoc 2007, 59:325-331.

9. Endo S, Suzuki Y, Takahashi G, Shozushima T, Ishikura H, Murai A, Nishida T, Irie Y, Miura M, Iguchi H, Fukui Y, Tanaka K, Nojima T, Okamura Y: Usefulness of presepsin in the diagnosis of sepsis in a multicenter prospective study. J Infect Chemother 2012, 18:891-897.

10. Levy MM, Fink MP, Marshall JC, Abraham E, Angus D, Cook D, Cohen J, Opal SM, Vincent JL, Ramsay G, International Sepsis Definitions Conference: SCCM/ESICM/ACCP/ATS/SIS international sepsis definitions conference. Intensive Care Med 2001, 2003(29):530-538.

11. Calandra T, Cohen J: The international sepsis forum consensus conference on definitions of infection in the intensive care unit. Crit Care Med 2005, 33:1538-1548.

12. Tunkel AR, Hartman BJ, Kaplan SL, Kaufman BA, Roos KL, Scheld WM Whitley RJ: Practice guidelines for the management of bacterial meningitis. Clin Infect Dis 2004, 39:1267-1284.

13. Kurihara T, Yanagida A, Yokoi H, Koyata A, Matsuya T, Ogawa J, Okamura Y, Miyamoto D: Evaluation of cardiac assays on a benchtop chemiluminescent enzyme immunoassay analyzer, PATHFAST. Anal Biochem 2008, 375:144-146.

14. Shapiro NI, Wolfe RE, Moore RB, Smith E, Burdick E, Bates DW: Mortality in emergency department sepsis (MEDS) scores: a prospectively derived and validated clinical prediction rule. Crit Care Med 2003, 31:670-675.

15. Knaus WA, Draper EA, Wagner DP, Zimmerman JE: APACHE Il:a severity of disease classification system. Crit Care Med 1985, 13:818-829.

16. Martin GS, Mannino DM, Eaton S, Moss M: The epidemiology of sepsis in the United States from 1979 through 2000. N Engl J Med 2003, 348:1546-1554.

17. Angus DC, Linde-Zwirble WT, Lidicker J, Clermont G, Carcillo J, Pinsky MR: Epidemiology of severe sepsis in the United States: analysis of incidence, outcome and associated costs of care. Crit Care Med 2001, 29:1303-1310

18. Mussap M, Noto A, Fravega M, Fanos V: Soluble CD14 subtype presepsin (sCD14-ST) and lipopolysaccharide binding protein (LBP) in neonatal sepsis: new clinical and analytical perspectives for two old biomarkers. J Matern Fetal Neonatal Med 2011, 24:12-14.

19. Naitoh K, Shirakawa K, Hirose J, Nakamura M, Takeuchi T, Hosaka Y, Furusako $\mathrm{S}$ : The new sepsis marker, SCD14-ST (PRESEPSIN): induction mechanism in the rabbit sepsis models. Crit Care 2010, 14:P19. 
20. Schuetz $P$, Christ-Crain M, Mueller B: Procalcitonin and other biomarkers for the assessment of disease severity and guidance of treatment in bacterial infections. Adv Sepsis 2008, 6:82-89.

21. Naeini $A E$, Montazerolghaem S: Procalcitonin marker for sepsis diagnosis. Evaluating a rapid immuno-chromatografi c test. Saudi Med J 2006, 27:422-424.

22. Oshita H, Sakurai J, Kamitsuna M: Semi-quantitative procalcitonin test for the diagnosis of bacterial infection: clinical use and experience in Japan. J Microbiol Immunol Infect 2010, 43:222-227.

23. Schuetz $P$, Chiappa V, Briel M, Greenwald JL: Procalcitonin algorithms for antibiotic therapy decisions. A systematic review of randomized controlled trials and recommendations for clinical algorithms. Arch Intern Med 2011, 171:1322-1331.

24. Schuetz P, Briel M, Christ-Crain M, Stolz D, Bouadma L, Wolff M, Luyt CE, Chastre J, Tubach F, Kristoffersen KB, Wei L, Burkhardt O, Welte T, Schroeder S, Nobre V, Tamm M, Bhatnagar N, Bucher HC, Mueller B: Procalcitonin to guide initiation and duration of antibiotic treatment in acute respiratory infections: an individual patient data meta-analysis. Clin Infect Dis 2012, 55:651-662.

25. Christ-Crain M, Müller B: Procalcitonin in bacterial infections - hype, hope, more or less? Swiss Med Wkly 2005, 135:451-456.

26. Venkataraman R, Kellum JA: Sepsis: update in the management. Adv Chronic Kidney Dis 2013, 20:6-13.

27. Dellinger RP, Levy MM, Rhodes A, Annane D, Gerlach H, Opal SM, Sevransky JE, Sprung CL, Douglas IS, Jaeschke R, Osborn TM, Nunnally ME, Townsend SR, Reinhart K, Kleinpell RM, Angus DC, Deutschman CS, Machado FR, Rubenfeld GD, Webb SA, Beale RJ, Vincent JL, Moreno R, Surviving Sepsis Campaign Guidelines Committee including the Pediatric Subgroup: Surviving Sepsis Campaign: international guidelines for management of severe sepsis and septic shock. Crit Care Med 2013, 41:580-637.

28. Ruiz-Alvarez MJ, García-Valdecasas S, de Pablo R, Sanchez García M, Coca C, Groeneveld TW, Roos A, Daha MR, Arribas I: Diagnostic efficacy and prognostic value of serum procalcitonin concentration in patients with suspected sepsis. J Intensive Care Med 2009, 24:63-71.

doi:10.1186/cc13070

Cite this article as: Liu et al:: Diagnostic value and prognostic evaluation of Presepsin for sepsis in an emergency department. Critical Care 2013 17:R244.

\section{Submit your next manuscript to BioMed Central and take full advantage of:}

- Convenient online submission

- Thorough peer review

- No space constraints or color figure charges

- Immediate publication on acceptance

- Inclusion in PubMed, CAS, Scopus and Google Scholar

- Research which is freely available for redistribution 